\title{
El estudio de la política subnacional en el Perú: posibilidades, avances y desafíos
}

\author{
JORGE ARAGÓN* \\ Pontificia Universidad Católica del Perú \\ jaragon@pucp.pe \\ MARÍA GRACIA BECERRA** \\ Pontificia Universidad Católica del Perú \\ mgbecerra@pucp.pe \\ https://doi.org/10.18800/rcpg.201602.001
}

\section{EL GIRO HACIA LO SUBNACIONAL EN LA POLÍTICA COMPARADA}

Durante las últimas décadas, la gran mayoría de Estados y sociedades latinoamericanos han transitado por procesos de reforma institucional y cambio estructural que han sido particularmente significativos. Sin duda alguna, y sobre todo dentro de los Estados unitarios de esta región, las experiencias de descentralización han sido uno de estos procesos. En esta dirección, como sostiene Falletti (2010), la transferencia de recursos, responsabilidades y autoridad de los niveles nacionales a los niveles regionales y locales de gobierno es uno de los cambios que mayor impacto ha tenido en los sistemas políticos latinoamericanos. Incluso en el Perú, donde esta reforma viene mostrando cierto estancamiento, es evidente que la descentralización ha revitalizado, en varios sentidos, las dinámicas políticas regionales y locales (Aragón e Incio, 2014).

$\mathrm{Si}$ a esto se le agrega el reconocimiento de que todo fenómeno y proceso político se despliegue de manera desigual a lo largo del territorio; y que este territorio incluye a autoridades, funcionarios públicos y ciudadanos vinculados a diferentes niveles de gobierno (nacional, regional y local); se hacen evidentes, en primer lugar, los límites de estudiar estos fenómenos y procesos solo desde

\footnotetext{
* Coordinador de la especialidad de Ciencia Política y Gobierno y profesor del Departamento de Ciencias Sociales de la PUCP.

** Licenciada en Ciencia Política y Gobierno y miembro del Grupo de Investigación de Política Subnacional de la Pontificia Universidad Católica del Perú.
} 
una perspectiva nacional. En segundo lugar, se hace evidente la necesidad de dar cuenta de las principales diferencias y similitudes en relación con las unidades territoriales y subnacionales involucradas en estos fenómenos o procesos políticos.

El desarrollo de este giro subnacional dentro de la política comparada alude a una estrategia de investigación que se centra en los actores, instituciones, estructuras y procesos que están localizados en unidades territoriales dentro de los países, esto es, por debajo de lo nacional e internacional (Giraudy, Snyder y Moncada, 2016). Cabe resaltar también que este enfoque subnacional contiene varias ventajas o fortalezas adicionales.

En esta dirección, Snyder (2009) plantea que un enfoque subnacional amplía y mejora el repertorio de posibilidades metodológicas con el que cuentan los investigadores. Por ejemplo, considerar a las unidades subnacionales es un modo eficaz de aumentar el número de observaciones en un diseño de investigación caracterizado por un $\mathrm{N}$ pequeño. Siempre y cuando no se pierda de vista que no se trata solo de dar cuenta de las relaciones existentes entre estas observaciones, sino también de incorporar en el análisis el hecho de que ellas, con mucha frecuencia, pertenecen a diferentes niveles en relación con lo nacional y subnacional.

También, según Snyder (2009), el enfoque subnacional permite fortalecer la capacidad de controlar por posibles variables explicativas, lo cual aumenta la posibilidad de obtener inferencias causales válidas en una investigación con $\mathrm{N}$ pequeño (Snyder, 2009). Por ejemplo, comparar dos unidades subnacionales de países contiguos aumenta significativamente la posibilidad de controlar los hallazgos por las condiciones culturales, históricas y socioeconómicas de estos países.

Pensando en los desafíos que implica clasificar y codificar diferentes casos, Snyder (2009) ańade que el enfoque subnacional modera algunos de los sesgos que afectan el tratamiento de los casos nacionales. Para comenzar, las investigaciones que abarcan distintos países suelen basarse, para la codificación de los casos de estudio, en medias subnacionales. Ello genera problemas importantes ya que, por ejemplo, muchos países a los que se les clasifica como semidesarrollados a partir de ciertos índices nacionales son, en realidad, una mezcla de sectores desarrollados y no desarrollados. En ese sentido, dos países con promedios nacionales muy similares pueden ser significativamente diferentes. De igual modo, el enfoque subnacional puede hacer evidente las situaciones en las que las características de una sola unidad subnacional sean consideradas de manera inapropiada como nacionales. 
Finalmente, en opinión de Snyder (2009), el enfoque subnacional ayuda a la construcción teórica porque nos permite examinar el contraste que existe entre los fenómenos políticos observados a nivel nacional y los observados a nivel subnacional. Lo que a su vez es clave para identificar los mecanismos por los cuales determinadas zonas o territorios, que son parte de un sistema político unificado, experimentan trayectorias muy distintas entre sí.

Ahora bien, el enfoque subnacional no está exento de algunas limitaciones. Entre otras cosas, Snyder (2009) menciona el hecho que la interdependencia, difusión y adopción de medidas tomadas en diferentes unidades subnacionales dentro de un mismo país limita la capacidad de hacer observaciones y pruebas independientes para cada hipótesis. La buena noticia es que este problema puede ser mitigado si se combinan las comparaciones realizadas en un mismo país con comparaciones realizadas en unidades subnacionales de distintos países.

$\mathrm{Si}$, como parece ser el caso, es mucho lo que se puede ganar a partir del desarrollo de una perspectiva o enfoque subnacional en el análisis de diferentes procesos y fenómenos políticos, una pregunta relevante es cuánto se ha avanzado en nuestro país en esta dirección. Como se verá más adelante, no se trata de un espacio vacío. Aunque, ciertamente, es todavía bastante menos si se compara con otros países de la región.

\section{Las investigaciones sobre política subnacional en el Perú}

Lo que hemos venido definiendo como política subnacional (léase, instituciones, autoridades, actores y procesos que existen y operan más allá del centro o la capital de un país) no es un fenómeno reciente en la historia política del Perú. Sin embargo, es un hecho que el último proceso de descentralización que se inició durante el gobierno de Alejandro Toledo - y que, como toda reforma de este tipo, consistió en la transferencia de competencias, recursos y responsabilidades desde la administración central hacia las autoridades regionales y locales - , ha sido un factor determinante para incentivar y revitalizar las dinámicas políticas regionales y locales en nuestro país. Como resultado de todos estos cambios, durante los últimos años, creció también el interés académico por lo que venía sucediendo en los ámbitos regional y local.

En un primer momento, las investigaciones sobre política subnacional en el Perú abordaron, sobre todo, la relación entre los partidos nacionales y las nuevas organizaciones políticas regionales y locales; y la naturaleza particular de los movimientos políticos regionales y de las organizaciones políticas locales 
que rápidamente empezaban a dominar la vida política en las regiones. No pasó mucho tiempo para que estuviera suficientemente estudiada y documentada la muy limitada presencia de los partidos políticos nacionales en las diferentes regiones del país y el hecho que la gran mayoría de organizaciones políticas a nivel regional enfrentaba los mismos desafíos que los partidos nacionales (Barrenechea, 2014; Remy, 2010; Vera, 2010). Es decir, la dificultad para atraer militantes, simpatizantes y electores, y, sobre todo, para mantenerlos en el tiempo. La ausencia de un mínimo de institucionalidad con relación a su organización interna. Y gestiones públicas con altos niveles de desaprobación. En esta línea, habría que destacar también los trabajos que encontraron que las dinámicas políticas subnacionales podían variar mucho de una región a otra (Becerra, Augusto, Retamozo y Ugaz, 2015; Aragón, 2014).

Este interés inicial por las organizaciones políticas formales en los ámbitos regionales, ha ido incorporando también algunas investigaciones sobre políticos y élites regionales. Con relación al primero de estos temas, ahora conocemos un poco más sobre las autoridades subnacionales que muestran interés por reelegirse en sus cargos (Córdova e Incio, 2013; Aragón e Incio, 2014). En cuanto a las élites regionales, Muñoz, Monsalve, Guibert, Guadalupe y Torres (2016) analizan cuatro regiones a profundidad (Arequipa, Cusco, Piura y San Martín) y encuentran que, en un contexto de debilidad de la institucionalidad política, la composición y funcionamiento de la estructura económica de cada departamento condiciona tanto el tipo de élite económica que tiende a desarrollarse como la dinámica política regional.

Como un punto de encuentro interesante entre el interés por organizaciones políticas y por actores políticos individuales en los ámbitos regionales, tenemos el trabajo de Zavaleta (2014), el mismo que describe y analiza a los políticos regionales como independientes en busca de coalición cada vez que hay elecciones para elegir gobiernos regionales y locales. Estas coaliciones de independientes suelen romperse pasado el periodo electoral, y esto sucede independientemente de que se pierda o se gane la elección.

En los últimos años, las investigaciones sobre política subnacional en el Perú han abordado nuevos temas. Por un lado, hay quienes se han dedicado a estudiar y discutir si lo que hemos visto durante los últimos ańos en nuestro país responde a la emergencia de regímenes autoritarios subnacionales o si, por el contrario, se trata más bien de prácticas autoritarias. En esta línea, por ejemplo, Cueva, Chávez, Encinas y Ramírez (2016) sostienen que en el caso de César Álvarez (Áncash), Iván Vásquez (Loreto), Gregorio Santos (Cajamarca) y Gerardo Viñas (Tumbes), lo que se observa es un sistemático abuso del poder; 
y plantean la necesidad de desarrollar una agenda de investigación que pueda explicar las causas y las consecuencias de estas variaciones territoriales.

Por otro lado, empieza a notarse también un mayor interés por la gestión pública regional y local (Ruiz, 2016; Salas, 2016; Manrique, 2016). En buena cuenta, se trata de analizar lo que vienen haciendo las autoridades regionales y locales para cumplir con las responsabilidades de gestión que les han sido asignadas; $y$ de recopilar la experiencia de funcionarios y asesores de las instituciones públicas regionales y locales para identificar problemas y soluciones de cara a la implementación de políticas públicas en ámbitos subnacionales.

\section{LOS TRABAJOS QUE APARECEN EN ESTE NÚMERO TEMÁTICO}

Los trabajos que componen este número temático de la Revista de Ciencia Política y Gobierno sobre política subnacional en el Perú han sido elaborados por miembros del Grupo de Investigación de Política Subnacional de la Pontificia Universidad Católica del Perú (GIPSN-PUCP) ${ }^{1}$. Este grupo de investigación está conformado por docentes, egresados de pre y posgrado, y estudiantes de Ciencia Política de la PUCP; y tiene como principal objetivo promover la investigación, el debate académico y la difusión de evidencia y conocimiento sobre política subnacional en el Perú.

En conjunto, se trata de un grupo de trabajos bastante recientes que se insertan en y dialogan con lo que ha sido la agenda de investigación que asume que los espacios subnacionales dentro de cualquier país tienen su propia dinámica política (Dosek, 2015, p. 17). Que aparezcan en una sola publicación responde a la apertura y acogida de Carlos Alza y Erick Mormontoy, editor y editor administrativo de esta revista, quienes nos dieron todas las facilidades para preparar este número temático.

Estos mismos trabajos pueden agruparse en tres grandes ejes: (1) regímenes, sistemas o dinámicas políticas subnacionales, (2) dinámicas electorales subnacionales y (3) ambición política en el nivel subnacional. Con relación al primero de estos ejes, tenemos dos artículos dedicados a discutir hasta qué punto y de qué manera se puede hablar en el Perú de regímenes, sistemas o dinámicas políticas a nivel subnacional. Así, mediante el estudio a profundidad de las regiones de Áncash y Loreto, Rosa Arévalo y Paolo Sosa analizan las condiciones que permitieron el surgimiento de dinámicas autoritarias en estas regiones. Por su

\footnotetext{
1 Se puede encontrar más información sobre este grupo de investigación en el siguiente enlace: http://investigacion. pucp.edu.pe/grupos/politica-subnacional/
} 
parte, Daniel Encinas aporta una serie de consideraciones teóricas a tener presente dentro de este debate y ofrece un conjunto de elementos a tener en cuenta para el desarrollo de una agenda de investigación sobre estos temas.

Dentro del segundo eje, dos artículos estudian algunas de las más importantes dinámicas electorales a nivel subnacional. Darío Pedraglio busca determinar qué factores influyeron en el desempeño electoral de los alcaldes y organizaciones que buscaron la reelección a nivel provincial en el Perú en el año 2010. Dada la fragilidad institucional de la gran mayoría de organizaciones políticas subnacionales, es sumamente sugerente el análisis por separado que este autor ofrece del desempeńo electoral de organizaciones y de candidatos. Por su lado, Mariana Ramírez estudia las revocatorias en América Latina, buscando identificar las condiciones que explican que efectivamente se activen o no procesos de revocatoria en grandes ciudades. Para ello, la autora realiza un análisis del nivel de dificultad para activar este mecanismo en cinco países de América Latina (Argentina, Colombia, Ecuador, Perú y Venezuela), para luego hacer un análisis a profundidad del caso de Lima Metropolitana.

Finalmente, dos artículos se centran en el estudio de la ambición política a nivel subnacional. Por su parte, José Luis Incio y Carmen Chavarría realizan un estudio descriptivo de los patrones de ambición de alcaldes y regidores distritales electos en las elecciones de 1980, 1993 y 2002. Este análisis amplía de manera significativa el rango temporal que suele primar en las investigaciones más recientes sobre política subnacional en el Perú; es decir, de 2002 en adelante. En una línea similar, Julio Gutiérrez analiza las trayectorias políticas de los candidatos que se presentaron en las elecciones del año 2014 a nivel provincial, identificando provincias con distintos patrones de construcción de carreras políticas. Para complementar su análisis, realiza también un análisis comparado y mucho más detallado de dos provincias: Satipo y Yungay.

¿En qué medida estos trabajos superan o no algunas de las limitaciones de lo que ha sido la literatura sobre política subnacional en el Perú?, ¿en qué medida ofrecen nuevas perspectivas y entradas de análisis?, o ¿en qué medida contribuyen a mejorar el conocimiento que actualmente tenemos sobre las dinámicas políticas subnacionales en nuestro país?, son preguntas que quedan por ahora sin responder. Sin embargo, estamos seguros que los autores de estos artículos estarán más que interesados en participar de estos debates. 


\section{BibLIOGRAFÍA}

Aragón, J. (2014). Política electoral subnacional: Fragmentación, competitividad y concentración regional, provincial y distrital. En A. M. Manrique y J. Zegarra (eds.), Cátedra Arequipa. Primera Sesión. Partidos politicos y movimientos regionales (pp. 73-101). Arequipa, Perú: Instituto Gobierno y Desarrollo Humano y Adrus D\&L Editores.

Aragón, Jorge e Incio, J. L. (2014). La reelección de autoridades regionales y municipales en el Perú, 2006-2014. Revista Argumentos, 5. Disponible en http://revistaargumentos.iep.org.pe/articulos/ la-reeleccion-de-autoridades-regionales-y-municipales-en-el-peru-2006-2014/

Barrenechea, R. (2014). Becas, bases y votos: Alianza para el Progreso y la política subnacional en el Perú. Lima, Perú: Instituto de Estudios Peruanos.

Becerra, M. G., Augusto, M. C., Retamozo, D. y Ugaz, S. (2015). El éxito de Francis Allison en el distrito de Magdalena del Mar (2002-2014). Un análisis empírico de la ventaja del incumbente a nivel subnacional. Revista de Ciencia Politica Politai, 6(10), 56-72.

Córdova, B. e Incio, J. L. (2013). La ventaja del incumbente en el ámbito subnacional: un análisis de las dos últimas elecciones municipales en el Perú. Papel Político, 18(2), 415-436.

Cueva, C., Chávez, N., Encinas, D. y Ramírez, M. (2016). Democracia y territorio en países unitarios: Una agenda pendiente. Lima, Perú: Jurado Nacional de Elecciones.

Dosek, T. (2015). Presentación. Revista de Ciencia Política Politai, 6(10), 7-8.

Falleti, T. (2010). Decentralization and Subnational Politics in Latin America. Nueva York, NY: Cambridge University Press.

Giraudy, A., Snyder, R. y Moncada, E. (2016). Subnational Research in Comparative Politics: Achievements and Future Directions. Manuscrito no publicado.

Manrique, A. M. (2016). Gobiernos regionales: entre la gestión y las políticas públicas. En A. M. Manrique, y J. Zegarra (eds.), Cátedra Arequipa. Tercera Sesión. Gestión y Politicas Públicas en Gobiernos Regionales (pp. 123-143). Arequipa, Perú: Instituto Gobierno y Desarrollo Humano y Adrus D\&L Editores.

Muñoz, P., Monsalve, M., Guibert, Y., Guadalupe, C. y Torres, J. (2016). Élites regionales en el Perú en un contexto de boom fiscal: Arequipa, Cusco, Piura y San Martín (2000-2013). Lima, Perú: Centro de Investigaciones de la Universidad del Pacífico.

Remy, M. I. (2010). Elecciones Regionales 2010 o el sueño de la candidatura propia. Revista Argumentos, 3. Disponible en http://revistaargumentos.iep.org.pe/articulos/ elecciones-regionales-2010-o-el-sueno-de-la-candidatura-propia/

Ruiz, M. (2016). Experiencias de gestión en un gobierno regional. En P. Sosa (ed.), Desde adentro: casos prácticos de gestión en el estado peruano (pp. 61-96). Lima, Perú: Instituto de Estudios Peruanos.

Salas, P. (2016). Políticas públicas en gobiernos subnacionales. En A. M. Manrique y J. Zegarra (eds.), Cátedra Arequipa. Tercera Sesión. Gestión y Políticas Públicas en Gobiernos Regionales (pp. 179-191). Arequipa, Perú: Instituto Gobierno y Desarrollo Humano y Adrus D\&L Editores.

Snyder, R. (2009). Reducción de la escala: el método comparativo de unidades subnacionales. Desarrollo Económico, 49(10), 287-309.

Vera, S. (2010). Radiografía a la política en las regiones: tendencias a partir de la evidencia de tres procesos electorales. Revista Argumentos, 5. Disponible en http://revistaargumentos.iep.org.pe/ articulos/radiografia-a-la-politica-en-las-regiones-tendencias-a-partir-de-la-evidencia-de-tresprocesos-electorales/ 